\title{
Benign Sphenoid Sinus Neoplasm
}

National Cancer Institute

\section{Source}

National Cancer Institute. Benign Sphenoid Sinus Neoplasm. NCI Thesaurus. Code C4422.

A non-metastasizing neoplasm that arises from the sphenoid sinus. 